\title{
Assessing the monitor warm-up time required before a psychological experiment can begin
}

\author{
Christian H. Poth ${ }^{\mathrm{a},}$ and Gernot Horstmann ${ }^{\mathrm{a}}$ \\ ${ }^{\mathrm{a}}$ Department of Psychology and Cluster of Excellence Cognitive Interaction Technology, Bielefeld University
}

\begin{abstract}
Visual experiments in psychology, psychophysics, and cognitive neuroscience require precise control over stimulus characteristics, such as luminance and contrast. In such experiments, stimuli are most often presented using computer monitors. These monitors bear an often neglected and rarely reported source of experimental confounds and uncontrolled variation: they take a warm-up time in which their luminance systematically changes until a stable level is eventually reached. Here we demonstrate this problem by measuring luminance over time for five different monitors. Results indicate that not only the warm-up time but also the course that the warm-up takes can vary greatly between different monitors. To address this problem, we propose a simple method of approximating a monitor's warm-up time, which takes into account theoretical considerations of the specific experiment. On this basis, we suggest a standardized experimental procedure and a standardized way of reporting its results to enable experimenters to control for monitor warm-up time.
\end{abstract}

Keywords $₫$ vision science, CRT, LED, luminance, contrast. Tools $\backsim$ R.

Acting Editor $\approx$ Denis Cousineau (Université d'Ottawa)

Reviewers

- One anonymous reviewer.

\section{c.poth@uni-bielefeld.de}

CHP: 0000-0003-1621-4911; GH: 0000-0002-2644-414X

10.20982/tqmp.13.3.p166

\section{Introduction}

Stimulus control is a corner stone of experimental psychology, psychophysics, and cognitive neuroscience. For instance, experimental psychologists and psychophysicists often investigate how visual perception changes as a function of carefully titrated stimulus manipulations (for overviews, see Kingdom \& Prins, 2010; Lu \& Dosher, 2013). This would be impossible without precise control over the intensity and other characteristics of visual stimuli. Nowadays, by far, most experimenters use computer monitors to present visual stimuli to their observers. This enables a computerized and thus fully-automated presentation of visual stimuli, which is more time-efficient, more flexible, and easier to replicate compared with classic mechanically-controlled presentation methods (such as the tachistoscope which required manual stimulus creation and presentation, e.g., Benschop, 1998, or mechanically clocked slide projectors).

Despite their advantages, however, computer monitors also have a number of limitations that must be taken into account before they can be used in psychological and neuroscientific experiments (Bauer, 2015; Elze \& Tanner, 2012; Klein, Zlatkova, Lauritzen, \& Pierscionek, 2013; Metha, Vingrys, \& Badcock, 1993). Doing so is complicated by the fact that the specific stimulus (Goris, Wagemans, \& Wichmann, 2008), the stimulus presentation software, the specific monitor technology (Foerster, Poth, Behler, Botsch, \& Schneider, 2016; Poth, Foerster, Behler, Schneider, \& Botsch, under review) and even the individual monitor (e.g. through aging) can suffer from their own particular set of limitations.

One limitation of computer monitors is often neglected in experimental procedures and also rarely reported: monitors need warm-up time during which their luminance changes systematically until it reaches stability of luminance (for one example reporting this warm-up time, however, see Petersen \& Andersen, 2012). Stimulus luminance directly affects basic visual processing and can thus have tremendous effects on stimulus visibility (e.g., Lu \& 
Dosher, 2013). Therefore, the systematic changes of monitor luminance before warm-up has been completed are a source of confounds or noise, which should be accounted for in experimental procedures.

Here, we demonstrate that different monitors can vary considerably in the warm-up time they require to reach stable luminance and also in the particular course the warm-up takes. We focus on cathode-ray tube (CRT) monitors, as these are often viewed as the gold-standard of presentation devices for their high temporal accuracy and precision (Ghodrati, Morris, \& Price, 2015). However, we also consider one light-emitting diode (LED) monitor for comparison. The differences that we observed in the warmup behavior between these monitors indicate that monitor warm-up time needs to be assessed for individual monitors and, importantly, should be reported. To implement these recommendations, we propose a simple method for estimating and a standardized protocol for reporting the required warm-up time of a monitor.

\section{Methods}

Monitor luminance was measured using the X-Rite i1 Pro (Grand Rapids, MI, USA) spectrophotometer, placed at about screen center.

The first monitor that was assessed was a Sony Trinitron Multiscan G420 CRT (Sony, Tokio, Japan). For this monitor, measurements were manually-timed by the experimenter with a countdown clock, whereby the spectrophotometer was sampled by the vputil software (VPixx Technologies, Saint-Bruno, QC, Canada) running on a notebook. Two manual measurement series were conducted. The extended cool-down measurement started after the monitor had been cooled down over night and one measurement was taken every minute. The 1-hr cool-down measurement started after the monitor had been cooled down for one hour and one measurement was taken every $30 \mathrm{~s}$. For both manual measurement series, a gray display was used that was calibrated to be half the luminance that the monitor could produce (after it has been warmed-up for over an hour).

Four other monitors were then assessed in an automated fashion, by controlling the spectrophotometer using the Pypixxlib-toolbox (VPixx Technologies, Saint-Bruno, QC, Canada) extension for Python (2.7, Python Software Foundation, Beaverton, OR, USA) within our own Python scripts. For these measurements, each monitor was connected to the same notebook that was controlling the measurements. For each monitor, measurements were taken from a full white and a mid-gray display that were shown using the Psychopy 2 extension (Peirce, 2007) for Python (2.7, Python Software Foundation, Beaverton, OR, USA) without gamma-calibration, after the monitor had been cooled down overnight. For these automated measurement series, one measurement was taken every $30 \mathrm{~s}$. Three CRT-monitors were used, the Belinea 103085 (Brunen IT Service $\mathrm{GmbH}$, Wittmund, Germany) and two different exemplars of the ViewSonic G90fB (ViewSonic, Brea, CA, USA). In addition, the LED-monitor Dell (Dell, Round Stock, TX, USA) was used for comparison to the CRT-monitors.

\section{Results}

\section{A simple method for approximating the warm-up time required for psychological experiments}

To be able to warrant the stability of luminance required for psychological experiments, it is necessary to know the warm-up time until stability is reached. Luminance stability is the time it takes from monitor power-up until no further changes in luminance will occur that can affect the experimental variables. In principle, approximating this warm-up time can be accomplished by measuring luminance over time, computing the rate of change, and then by taking the point in time where the absolute rate of change falls below a certain threshold. However, this method does not apply to cases in which monitor warm-up takes a complex course, comprising initial fluctuations, increases to a maximum, and decreases of luminance (see the results of our manual measurements below). The absolute rate of luminance change over time is zero at a maximum, but increases afterwards when luminance starts to decrease over time. Therefore, the required warm-up time cannot be approximated as the time when the absolute rate of luminance change falls below a threshold. Instead, it has to be considered that this rate stays below the threshold as well.

We propose a simple method of approximating the warm-up time required for psychological experiments, which allows for complex warm-up behavior. The method is based on luminance measurements that are repeated for an extended time period. First, a threshold for the rate of change of luminance over time is assumed. This assumption has to be grounded on theoretical considerations: it should be the value of luminance per unit time for which no effect on experimental variables should occur. Second, a critical duration is assumed, after whose passing, it can safely be assumed that luminance will not change its function of time any more in terms of sign. Third, the luminance measurements are assessed as a function of time and fit with a smoothing spline, whose first derivative is used to approximate the rate of luminance change over time. Forth, the warm-up time is approximated as the time of the first measurement for which the absolute rate of luminance change is below the threshold, given it remains below threshold for the critical duration afterwards. List- 
Listing 1 a Function for approximating the required monitor warm-up time using R.

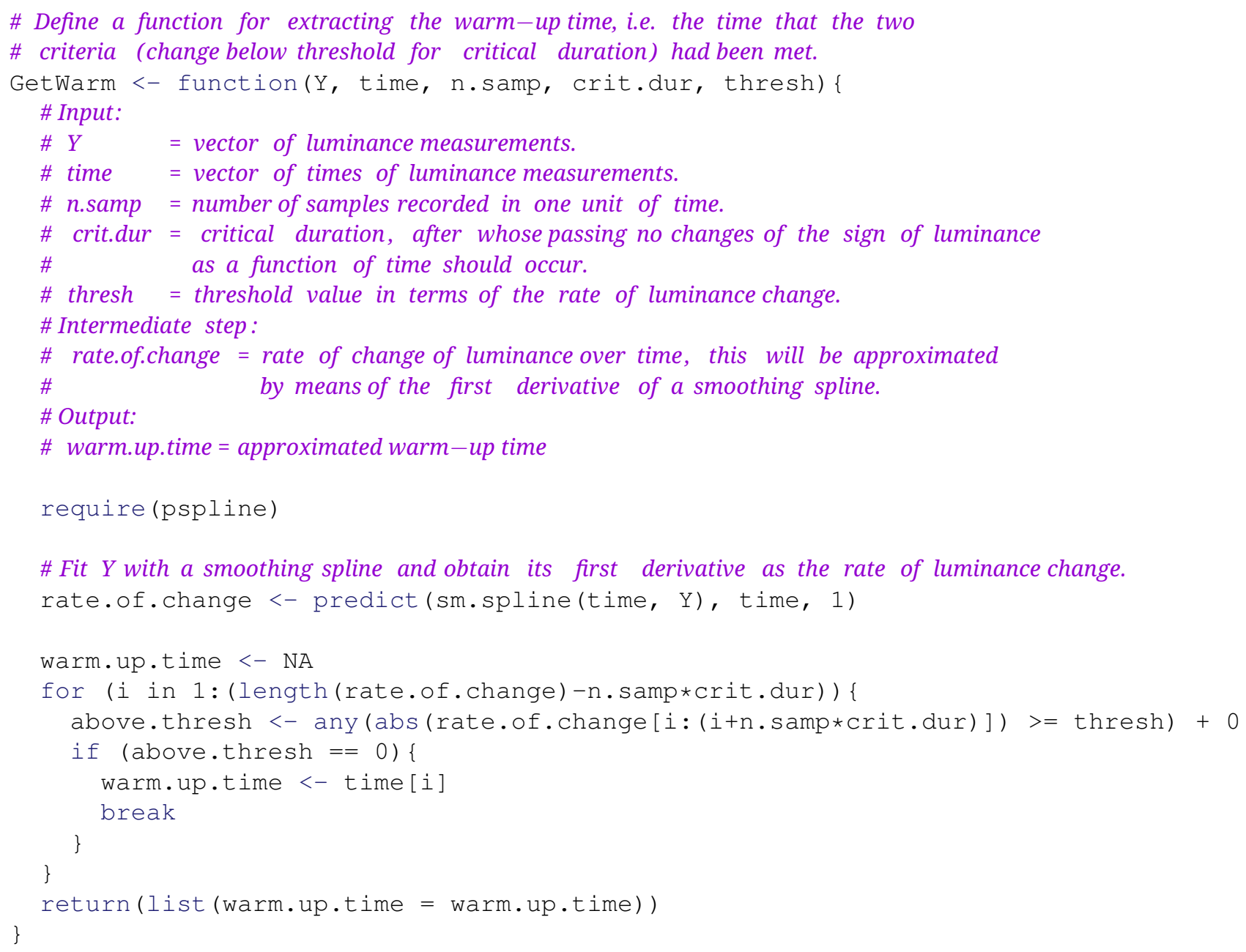

ing 1 provides an example script implementing the method in the R programming language (R Core Team, 2015; Ramsey \& Ripley, 2017). Listing 2 demonstrates how the method can be used by applying it to the data of the extended cooldown measurement series (see below).

\section{Manual measurements of warm-up behavior of one CRT-monitor}

Figure 1 depicts the results of the manual measurement series. Luminance increased in the first few minutes after monitor power-up. However, after reaching a maximum, luminance decreased until it reached a stable level. We approximated the required warm-up time for a psychological experiment by using the above-described method with a threshold of $0.5 \mathrm{~cd} / \mathrm{m}^{2}$ per minute and a critical duration of 3 minutes. Thus, we assumed that a temporal stability of luminance has been reached once the absolute rate of luminance change over time has remained below
$0.5 \mathrm{~cd} / \mathrm{m}^{2}$ for at least 3 minutes. This yielded an approximate required warm-up time of $20 \mathrm{~min}$ for the extended cool-down measurement series, which took place after the monitor cooled down overnight. For the 1-hr cool-down measurement series, the approximate warm-up time was $19 \mathrm{~min}$.

\section{Automated measurements of the warm-up behavior of three CRT-monitors}

Figure 2 depicts the results of the automated measurements of the warm-up behavior of the three other CRTmonitors, after they cooled-down overnight. In contrast to the previous CRT-monitor, the approximated warm-up times were much shorter for these three monitors. For these monitors, luminance over time was measured for a full white and a mid-gray display (without gamma linearization, see the Methods). For each monitor, both of these measurement series yielded comparable warm-up 
Listing 2 - Example script demonstrating the use of the function in Listing 1 . The data stored in vectors "Y" (luminance in $\mathrm{cd} / \mathrm{m}^{2}$ ) and "time" (time of luminance measurements) comes from the extended cool-down measurement series (see the Results section below).

\# Define some input parameters for the function GetWarm of Listing 1.

\# Define threshold luminance.

thresh $<-0.5$ \# i.e. $0.5 \mathrm{~cd} / \mathrm{m}^{2}$ per minute.

\# Define critical duration.

crit.dur <- 3 \#i.e. 3 minutes.

\# Indicate how many measurements there are for each unit time.

n. samp <- 1 \# here, 1 measurement per minute.

\# Data from the manual measurement series 1.

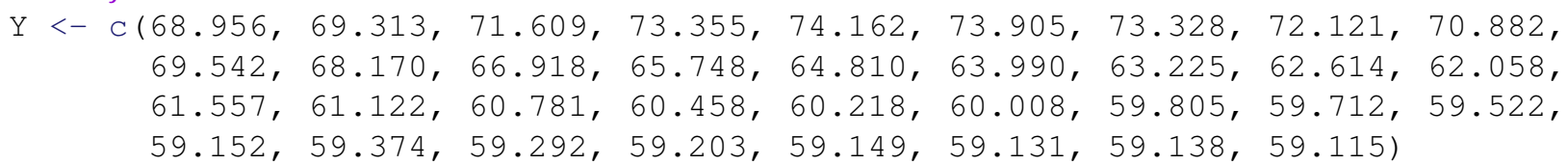

time $<-c(2,3,4,5,6,7,8,9,10,11,12,13,14,15,16,17,18,19$, $20,21,22,23,24,25,26,27,28,29,30,31,32,33,34,35,36)$

\# Apply the function to obtain the warm-up time according to the above defined criteria.

GetWarm(Y, time, n.samp, crit.dur, thresh)

\#\# Loading required package: pspline

\#\# \$warm.up.time

\#\# [1] 20

times. That is, the warm-up times for the full white and mid-gray displays were 3.47 and $3.61 \mathrm{~min}$ for the Belinea 103085, 2.90 and $2.98 \mathrm{~min}$ for the first ViewSonic G90fB, and 1.82 and $2.34 \mathrm{~min}$ for the second ViewSonic G90fB.

\section{Automated measurements of the warm-up behavior of the LED-monitor}

In addition to the CRT-monitors, we assessed the warm-up behavior of an LED-monitor, the Dell U2412M. Overall, the approximated warm-up time was shorter than for the CRTmonitor that we assessed first but not than the ones we assessed later (Figure 3). Interestingly, for the LED-monitor the warm-up time depended on the visual stimulus. That is, the warm-up time was approximated to be $9.17 \mathrm{~min}$ for the full white display but $0.34 \mathrm{~min}$ for the mid-gray one.

\section{Discussion}

We demonstrated that different monitors can vary greatly in warm-up times, the times that they need to reach stable luminance. Moreover, we found that the time course that the warm-up takes can vary between monitors. Both of these findings implicate the warm-up time of monitors as a potential source of noise or even confound in visual exper- iments of psychology and cognitive neuroscience. To help experimenters to control for such compromises, we proposed a simple method approximating the warm-up time a monitor (and stimulus) requires for running a specific psychological experiment.

By using a convenience sample of 4 CRT and 1 LEDmonitor, our measurement series show that the differences between monitor's warm-up times can be large, but that this does not have to be always the case. While for one CRT-monitor the warm-up time was about $20 \mathrm{~min}$, for three other monitors of two different monitor models it was about $3 \mathrm{~min}$. This was the case irrespective of the visual stimulus whose luminance was measured over time. In contrast, measurements of an LED-monitor yielded strikingly different warm-up times for a full white compared with a mid-gray display, revealing a dependency of warmup time on the particular stimulus.

Taken together, our observations indicate that there can be large differences in the warm-up behavior of monitors. This needs to be taken into account in laboratory procedures because collecting data before monitor warm-up is complete implies an ongoing luminance variation that may compromise experimental results. For ex- 
Figure 1 a Manual measurements of luminance over time of a CRT-monitor (Sony Trinitron Multiscan G420). The monitor showed a gray display calibrated to half the maximum luminance. The extended measurement series started after the monitor cooled down overnight, the 1-hr measurement series after it cooled down for one hour. Lines indicate smoothing splines. Vertical lines indicate the required warm-up time, as approximated by the above described method.

\section{Warm-up behavior of a CRT (calibrated mid-gray display)}

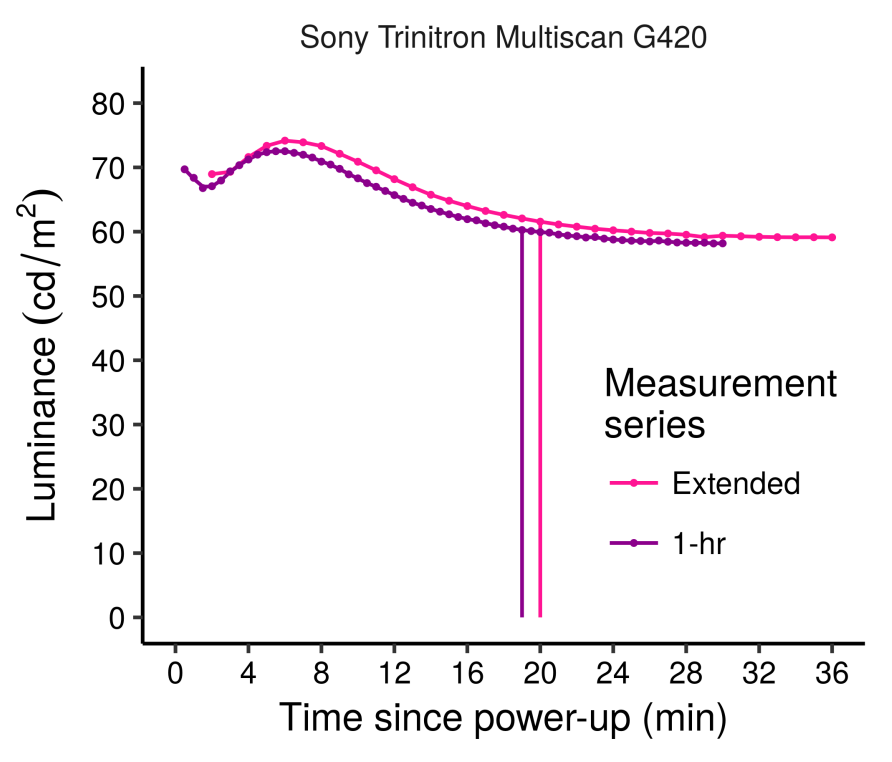

ample, it is common to first adaptively adjust the stimulus contrast to prevent floor or ceiling effects in a subsequent experiment. This procedure would fail, if systematic luminance variations occurred during the adjustment. The problem seems especially pressing in experiments on basic vision and elementary visual cognition, where stimulus luminance and contrast can have tremendous effects on performance in tasks such as object recognition pattern masking (e. g., Petersen \& Andersen, 2012; Poth, Herwig, \& Schneider, 2015; Poth \& Schneider, 2016). How sensitive a particular experiment will be to confounds by systematic luminance variations during monitor warmup has to be determined based on the experimenter's background knowledge. While experiments on visual processing may be greatly affected by stimulus contrast (Petersen \& Andersen, 2012), this need not be the case for experiments on cognition at higher levels of the processing hierarchy.
For example, it is common to first adaptively adjust the stimulus contrast to prevent floor or ceiling effects in a subsequent experiment. This procedure would fail, if systematic luminance variations occurred during the adjustment.
For experiments in which visual stimulus factors may play a role, we therefore propose that experimenter adopt the following general experimental procedures and reporting standard:
1. While conceiving an experiment, experimenters determine, based on theoretical considerations, how small a luminance change has to be to be safely assumed ineffective regarding the experimental variables. This luminance change can be used as threshold value for the abovedescribed method.

2. Based on the characteristics of their monitor, experimenters set a critical duration. After the absolute rate of luminance change has remained below threshold for this critical duration, it has to be assumable that no threshold will occur. This can later be checked against the data from luminance measurements over time. 
Figure 2 - Automated measurements of luminance over time for three CRT-monitors (Belinea 103085, ViewSonic G90fB 1, and 2). The monitors showed a full white (plotted in light blue) or mid-gray (plotted in dark blue) display. Lines indicate smoothing splines. Vertical lines indicate the required warm-up times, as approximated by the above described method.

\section{Warm-up behavior of different CRT-monitors}

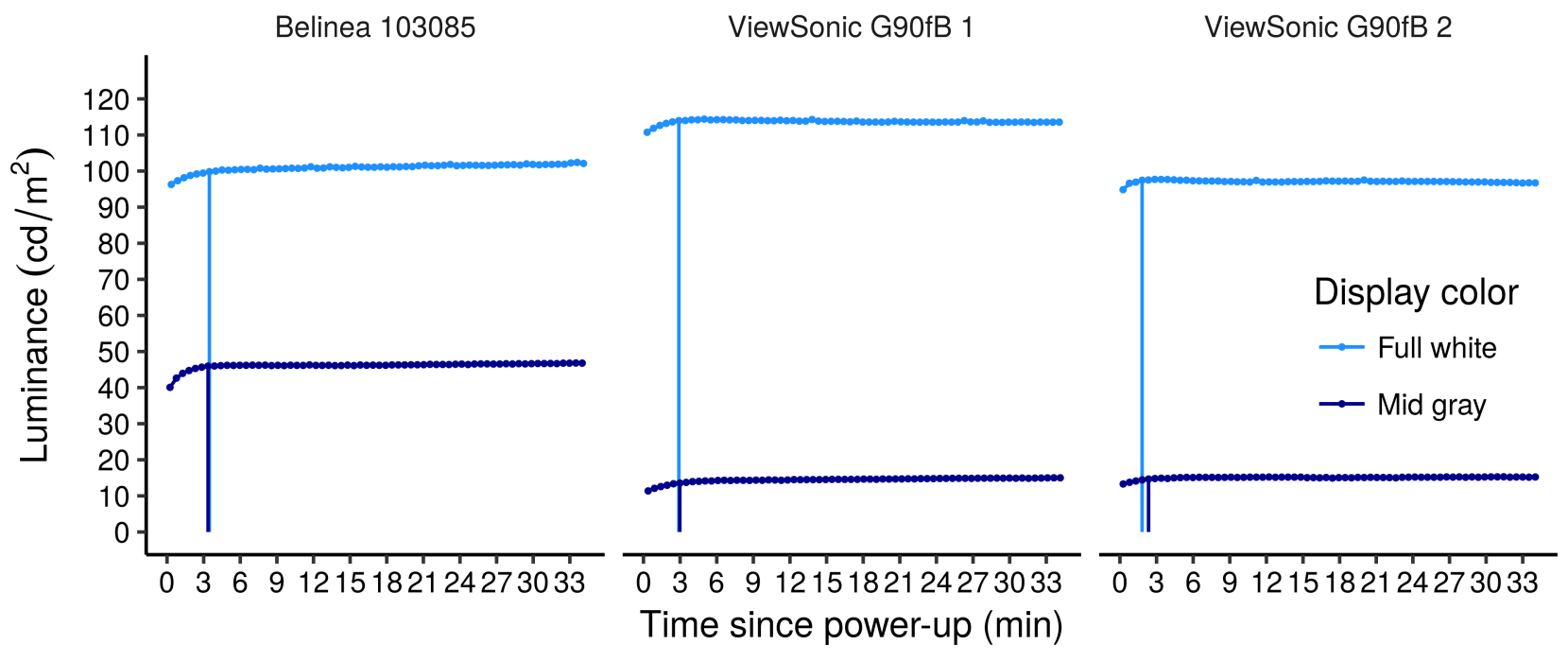

3. A visual stimulus that corresponds to the stimuli used in the experiment is displayed on the monitor and its luminance should be measured over time.

4. The threshold and critical duration are applied to the above-described method to process the measurement results, in order to approximate the warm-up time of the monitor and stimulus for the given criteria.

5. Before running the experiment, the monitor is warmed-up for the approximated warm-up time at least.

6. Within the description of the experimental procedures, experimenters state how the warm-up time was approximated, which criteria were used for this purpose, and that the monitor was indeed warmed up for this duration before the experiment. For example, referring to the above-proposed method, authors could state: "Before the experiment, the monitor was warmed up for at least $20 \mathrm{~min}$. This was the warmup time necessary for stability of luminance (approximated based on 35 luminance measurements with 1 measurement per min, using a rate of change threshold of $0.5 \mathrm{~cd} / \mathrm{m}^{2}$ per min and a critical duration of $3 \mathrm{~min}$."

\section{Authors' note}

Correspondence concerning this article should be addressed to Christian H. Poth, Department of Psychology and Cluster of Excellence Cognitive Interaction Technology, Bielefeld University, P.O. box 1001 31, 33501 Bielefeld, Germany. This work was supported by the Cluster of Excellence Cognitive Interaction Technology 'CITEC' (EXC 277) at Bielefeld University, which is funded by the German Research Foundation (DFG). Author contributions: CHP designed the work, performed the measurements, analyzed the data, programmed the approximation of warmup times, produced the figures, and wrote the paper. CHP and GH did the programming for the automated measurements.

\section{References}

Bauer, B. (2015). A timely reminder about stimulus display times and other presentation parameters on CRTs and newer technologies. Canadian Journal of Experimental Psychology/Revue Canadienne de Psychologie Expérimentale, 69(3), 264-273. doi:10.1037/ cep0000043

Benschop, R. (1998). What is a tachistoscope? Historical explorations of an instrument. Science in Context, 11(1). doi:10.1017/S0269889700002908

Elze, T. \& Tanner, T. G. (2012). Temporal properties of liquid crystal displays: Implications for vision science experiments. PLOS ONE, 7(9), e44048. doi:10.1371/journal. pone. 0044048

The Quantitative Methods for Psychology 
Figure 3 - Automated measurements of luminance over time of an LED-monitor (Dell U2412M). The monitor showed a full white (plotted in light blue) or mid-gray (plotted in dark blue) display. Lines represent smoothing splines. Vertical lines indicate the required warm-up times, as approximated by the above described method.

\section{Warm-up behavior of an LED-monitor}

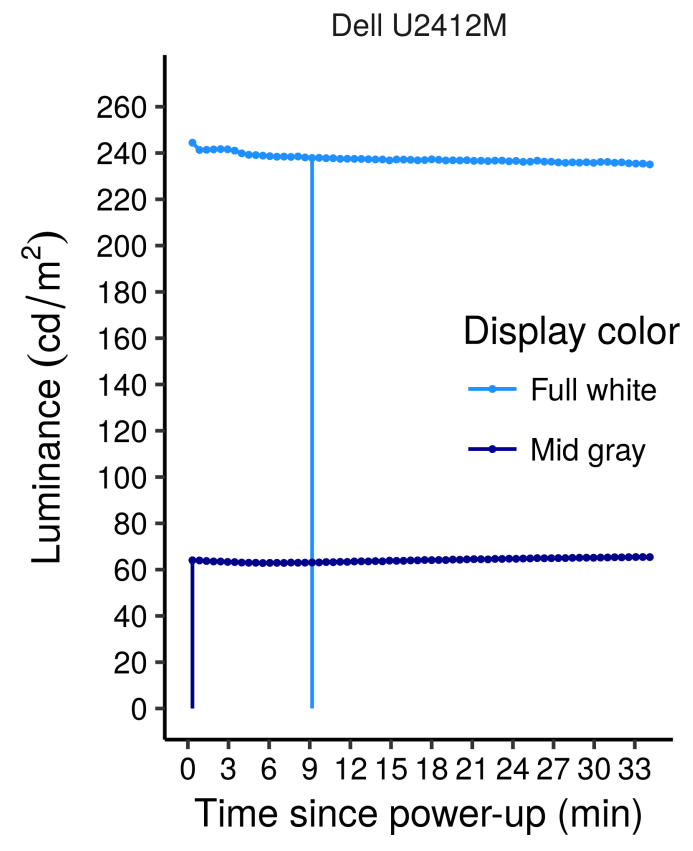

Foerster, R. M., Poth, C. H., Behler, C., Botsch, M., \& Schneider, W. X. (2016). Using the virtual reality device oculus rift for neuropsychological assessment of visual processing capabilities. Scientific Reports, 6, 1. doi:10. 1038/srep37016

Ghodrati, M., Morris, A. P., \& Price, N. S. C. (2015). The (un)suitability of modern liquid crystal displays (LCDs) for vision research. Frontiers in Psychology, 6. doi:10.3389/fpsyg.2015.00303

Goris, R. L. T., Wagemans, J., \& Wichmann, F. A. (2008). Modelling contrast discrimination data suggest both the pedestal effect and stochastic resonance to be caused by the same mechanism. Journal of Vision, 8(15), 17-17. doi:10.1167/8.15.17

Kingdom, F. A. A. \& Prins, N. (2010). Psychophysics: a practical introduction. London, UK: Academic Press.

Klein, J., Zlatkova, M., Lauritzen, J., \& Pierscionek, B. (2013). Photometric and colorimetric measurements of CRT and TFT monitors for vision research. Journal of Modern Optics, 60(14), 1159-1166. doi:10.1080/09500340. 2013.808385
Lu, Z.-l. \& Dosher, B. (2013). Visual psychophysics: from laboratory to theory. Cambridge, MA, USA: MIT Press.

Metha, A. B., Vingrys, A. J., \& Badcock, D. R. (1993). Calibration of a color monitor for visual psychophysics. $B e-$ havior Research Methods, Instruments, \& Computers, 25(3), 371-383. doi:10.3758/BF03204528

Peirce, J. W. (2007). Psychopy - Psychophysics software in Python. Journal of Neuroscience Methods, 162(1), 8-13. doi:10.1016/j.jneumeth.2006.11.017

Petersen, A. \& Andersen, T. S. (2012). The effect of exposure duration on visual character identification in single, whole, and partial report. Journal of Experimental Psychology: Human Perception and Performance, 38(2), 498-514. doi:10.1037/a0026728

Poth, C. H., Foerster, R. M., Behler, C., Schneider, W. X., \& Botsch, M. (under review). Ultra-high temporal resolution of visual presentation using gaming monitors and g-sync.

Poth, C. H., Herwig, A., \& Schneider, W. X. (2015). Breaking object correspondence across saccadic eye movements deteriorates object recognition. Frontiers in 
Systems Neuroscience, 9. doi:10 . 3389 / fnsys . 2015. 00176

Poth, C. H. \& Schneider, W. X. (2016). Breaking object correspondence across saccades impairs object recognition: The role of color and luminance. Journal of Vision, 16(11), 1. doi:10.1167/16.11.1
R Core Team. (2015). R: A language and environment for statistical computing. Vienna, Austria: R Foundation for Statistical Computing. Retrieved from https://www.Rproject.org/

Ramsey, J. \& Ripley, B. (2017). Pspline: Penalized Smoothing Splines (Version 1.0). Retrieved from https://CRAN.Rproject.org/package=pspline

\section{Citation}

Poth, C. H. \& Horstmann, G. (2017). Assessing the monitor warm-up time required before a psychological experiment can begin. The Quantitative Methods for Psychology, 13(3), 166-173. doi:10.20982/tqmp.13.3.p166

Copyright @ 2017, Poth and Horstmann. This is an open-access article distributed under the terms of the Creative Commons Attribution License (CC BY). The use, distribution or reproduction in other forums is permitted, provided the original author(s) or licensor are credited and that the original publication in this journal is cited, in accordance with accepted academic practice. No use, distribution or reproduction is permitted which does not comply with these terms.

Received: 07/07/2017 Accepted: 11/07/2016 